\title{
Quantitative Analysis of Pulmonary Emphysema using Isotropic Gaussian Markov Random Fields
}

\author{
Chathurika Dharmagunawardhana ${ }^{1}$, Sasan Mahmoodi ${ }^{1}$, Michael Bennett ${ }^{2}$ and Mahesan Niranjan ${ }^{1}$ \\ ${ }^{1}$ School of Electronics and Computer Science, University of Southampton, Southampton, SO17 1BJ, U.K. \\ ${ }^{2}$ National Institute for Health Research, Southampton Respiratory Biomedical Research Unit, University Hospital \\ Southampton NHS Foundation Trust, Tremona Road, Southampton, U.K. \\ $\{c d 6 g 10$, sm3,mn\}@ecs.soton.ac.uk,michaelbennett@soton.ac.uk
}

Keywords: Emphysema, Spatially Varying Parameters, Gaussian Markov Random Fields, Tissue Classification.

\begin{abstract}
A novel texture feature based on isotropic Gaussian Markov random fields is proposed for diagnosis and quantification of emphysema and its subtypes. Spatially varying parameters of isotropic Gaussian Markov random fields are estimated and their local distributions constructed using normalized histograms are used as effective texture features. These features integrate the essence of both statistical and structural properties of the texture. Isotropic Gaussian Markov Random Field parameter estimation is computationally efficient than the methods using other MRF models and is suitable for classification of emphysema and its subtypes. Results show that the novel texture features can perform well in discriminating different lung tissues, giving comparative results with the current state of the art texture based emphysema quantification. Furthermore supervised lung parenchyma tissue segmentation is carried out and the effective pathology extents and successful tissue quantification are achieved.
\end{abstract}

\section{INTRODUCTION}

Emphysema is a critical lung disease causing extensive lung tissue destruction and is currently emerging as a worldwide health problem. The main causes of emphysema are smoking or inhaling toxic air and a genetic disorder known as alpa-1-antitrypsin deficiency. The pulmonary function test (PFT) also known as spirometry and computed tomography (CT) analysis are the two common approaches used to diagnose emphysema. PFT is a clinical test associated with measuring breath volumes. High resolution CT (HRCT) image analysis is more informative than PFT and useful for analyzing pathology distribution, assessing severity and identifying a wide variety of sub type patterns of pulmonary lung diseases.

The amount of information present in CT imaging have largely increased with the recent technical developments of medical equipment. Therefore computer aided diagnosis (CAD) of CT scans is widely preferred (Litmanovich et al., 2009). Partially or fully automated CAD methods are fast, precise and highly reproducible compared to subjective visual assessment in medical image processing (Madani et al., 2001). CAD of emphysema mainly depends on texture features and intensity information.
In this paper we propose a new texture feature for emphysema quantification based on isotropic Gaussian Markov random fields (IGMRF). In Markov random field (MRF) feature extraction, the parameters of MRF model are used as texture features. The application of model based statistical features to emphysema quantification is not commonly used because of the relatively high computational cost. Also the model parameters are spatially constant, therefore, subtle pattern variations which are crucial in tissue discrimination are smoothen out.

However, the parameter estimation of IGMRFs is simple and fast compared to other MRFs and ideally suited for isotropic texture representation. In addition, we formulate the novel texture feature based on IGMRF characterized by spatially varying parameters addressing above issues in model based feature extraction. Spatially varying parameter estimation is closely related to the structural feature extraction. Therefore, the method itself is a semi parametric method and integrates the best of both statistical and structural features.

In this study, distributions of spatially varying parameters of IGMRFs constructed by normalized histograms are used as a discriminative texture feature. The approach is used to classify between healthy 
lung tissue (NT) and two sub types of emphysema, namely centrilobular emphysema (CLE) and paraseptal emphysema (PSE). The experimental results show that normal lung and emphysema, including its subtypes, can be efficiently discriminated using IGMRF features. The results are also compared against the state of the art LBP based emphysema quantification (Sørensen et al., 2010) and comparable performances are achieved.

We also perform supervised lung tissue segmentation on a CT slice dataset (Sørensen et al., 2013) and achieve satisfactory lung parenchyma pathology distributions and quantification of emphysema and its subtypes. The quantitative results correlates with the available labellings of visual inspection by experienced radiologists and emphysema index (EI). The novel texture features successfully discriminate the healthy lung tissue from emphysema and can be used in sub type diagnosis.

The remainder of this paper is organized as follows. In section 2 currently used methods of emphysema quantification are discussed. IGMRF model is introduced in section 3 and texture feature formulation is explained in section 4. Experimental results and discussions are elaborated in section 5 and finally, conclusions are given in section 6 .

\section{BACKGROUND}

CAD approaches based on lung CT scans for emphysema quantification can be categorized into intensity (CT density) based methods and texture based techniques. Under intensity based techniques, many studies have been carried out to find an absolute threshold value below which emphysema is considered to be present. (Muller et al., 1988) introduced a method known as 'emphysema index' or 'density mask' which measures the relative amount of lung parenchymal pixels that have attenuation values below -910 HU. Further studies have obtained the optimal threshold value for emphysema quantification as -950HU (Litmanovich et al., 2009). An adaptive thresholding method that also incorporates the information of pixel locations on the lung has been introduced by (Hara et al., 2004). Another approach has been proposed based on mean lung density and voxel dimensions (Coxson et al., 1999).

Intensity based CAD techniques usually suffer from problems caused by monotonic intensity changes. This may occur due to several reasons, for example, the influence of contrast materials and degree of inspiration of the lung while scanning (Muller et al., 1988). Secondly there is a degree of corruption in the CT image due to noise, arising from strict limitations on radiation power that can be applied on a patient (Sprawls, 1995). Techniques solely based on intensity are highly sensitive to the noise. (Häme et al., 2013) proposed a hidden Markov measure field model to obtain more promising emphysema index measures than standard densitometric approaches showing robustness to noise resulting from reconstruction kernels. However, intensity and texture based integrated approaches are the better choice for pulmonary lung disease analysis using CT (Sørensen et al., 2010).

Texture based CAD techniques are a successful methodology to use in assessing the presence and distribution of emphysema and its subtype patterns. The texture features can be categorized as statistical, spectral and structural features (Litmanovich et al., 2009). (Uppaluri et al., 1999) used a method known as adaptive multiple feature method (AMFM) which assessed 22 independent statistical features in order to classify different lung tissue patterns. This approach is further improved by extending it from $2 \mathrm{D}$ to 3D by (Xu et al., 2006), to classify emphysema and early smokingrelated pathologies. (Mishima et al., 1999) attempt to detect early emphysema on the basis of fractal analysis. Spatial gray level dependence method (SGLDM), Gray level run length method (GLRLM) and Gray level Difference Method (GLDM) have also been used as statistical feature extraction methods for emphysema diagnosis (Vasconcelos et al., 2010). (Depeursinge et al., 2010) have used the density histogram and quincunx wavelet frame coefficients with number of pixels below $-1000 \mathrm{HU}$ as the feature set to evaluate emphysema classification performance with different classifiers. In (Kim et al., 2009) statistical texture features as well as shape features are also employed in lung disease classification.

(Sluimer et al., 2003) have used Gaussian, Laplacian of Gaussian and first and second order derivatives of Gaussian filters in different scales and have obtained the histogram of responses as spectral texture features. (Depeursinge et al., 2007) have used discrete wavelet frame (DWF) to classify lung tissue types including emphysema. However, filter based methods require selection of an optimal filter set and employing reasonable sizes for filter kernels.

Structural features encapsulate information on structures of the texture such as arrangements of texture primitives. A texton based method has been introduced in (Gangeh et al., 2010). (Sørensen et al., 2010) applied rotational invariant local binary patterns (LBP) for successful emphysema quantification. This method acquires very compelling results and can be considered as the current state of the art texture based emphysema quantification method. 
In the present study we integrate the essence of statistical and structural properties of texture by formulating a hybrid feature extraction scheme. Therefore, these features can encapsulate the information about variations in the texture primitive and its arrangement patterns and also information about statistical dependencies and pixel interactions. IGMRF model is used to capture neighbor dependencies and spatially varying parameter estimation is used to capture structural information.

\section{IGMRF MODEL}

Gaussian Markov Random Field (GMRF) is an important subclass of MRFs and gives simplified ways of parameter estimation with less computational burden compared to that of MRFs (Rue and Held, 2005; Manjunath and Chellappa, 1991; Mahmoodi and Gunn, 2011). A local conditional probability distribution of GMRF model encapsulates spatial dependencies between a pixel and its neighbors, associating them in a Gaussian functional form (Petrou and Sevilla, 2006). Let $\Omega=\{(i, j) \mid 1 \leq i \leq H, 1 \leq j \leq W\}$ represent the set of grid points on a $H \times W$ regular lattice corresponding to the pixels in an image region. The image region on $\Omega$ is preprocessed to have zero mean. The intensity value of the pixel at $s=(i, j)$ position is given by $y_{s}$. The local conditional model of GMRF describing probability of $y_{s}$ given its neighbors $y_{s+r}, r$ being relative neighbor position on the neighborhood $\tilde{N}_{S}$ is given by,

$$
\begin{aligned}
& p\left(y_{s} \mid y_{s+r}, \alpha_{r}, \sigma, j \in \tilde{N}_{s}\right)= \\
& \frac{1}{\sqrt{2 \pi \sigma^{2}}} \exp \left\{-\frac{1}{2 \sigma^{2}}\left(y_{s}-\sum_{r \in \tilde{N}_{s}} \alpha_{r} \bar{y}_{s+r}\right)^{2}\right\}
\end{aligned}
$$

where $\alpha_{r}$ is the interaction coefficient which measures the influence by a neighbor intensity value at the neighbor position $r$. The neighbor pixels in symmetric positions about the considered pixel are assumed to have identical parameters, i.e. $\alpha_{r}=\alpha_{-r}$ (Petrou and Sevilla, 2006), therefore $\bar{y}_{s+r}=\left(y_{s+r}+y_{s-r}\right)$, the sum of two neighbor values situated in symmetric neighbor positions with respect to the pixel. Then $\tilde{N}_{s}$, is the asymmetric neighborhood such that if $r \in \tilde{N}_{s}$, then $-r \notin \tilde{N}_{s}$ (Zhao et al., 2007).

IGMRF is a special case of GMRF also known as circular symmetric GMRF and further simplify the GMRF model (Kashyap and Khotanzad, 1986). IGMRFs model non directional isotropic textures in a simplified rotational invariant framework with only two model parameters. Parameter estimation is simple and fast compared to other MRF models because

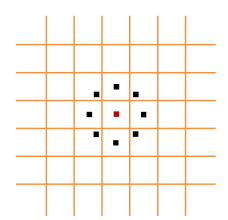

$(R, P)=(1,8)$

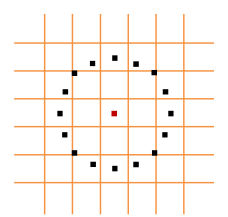

$(R, P)=(2,16)$

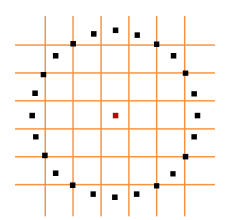

$(R, P)=(3,24)$
Figure 1: Multiresolution circular neighborhood system corresponding to $(R, P)=\{(1,8),(2,16),(3,24)\}$.

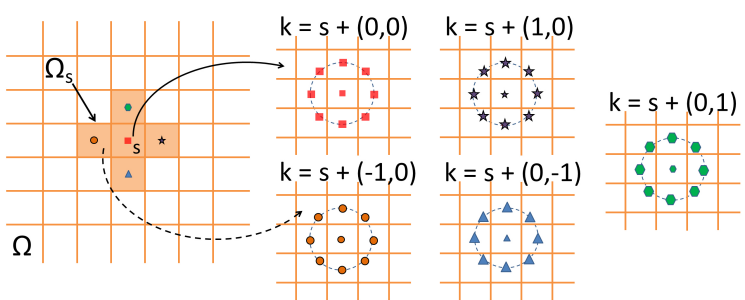

Figure 2: The five nearest neighborhood samples $N_{k}(k \in$ $\left.\Omega_{s}\right)$ corresponding to $(R, P)=(1,8)$ at pixel $s$ which is used for spatially varying parameter estimation.

solutions for parameters can be found analytically and without requiring any matrix inversion. IGMRF model is given by,

$$
\begin{aligned}
& p\left(y_{s} \mid y_{s+r}, \alpha, \sigma, r \in N_{s}\right)= \\
& \frac{1}{\sqrt{2 \pi \sigma^{2}}} \exp \left\{-\frac{1}{2 \sigma^{2}}\left(y_{s}-\alpha \sum_{r \in N_{s}} y_{s+r}\right)^{2}\right\}
\end{aligned}
$$

$\alpha$ and $\sigma$ are the model parameters to be estimated. $y_{s+r}$ are the neighbors in the circular symmetric neighborhood of $y_{s}$ and $N_{s}=\left\{r \mid r \in \tilde{N}_{s}\right\} \cup\{r \mid-r \in$ $\left.\tilde{N}_{s}\right\}$. Circular neighborhoods used in this study is shown in figure $1 . R$ is the radius and $P$ is the number of neighbors in a circle of radius $R$. This representation is similar to the neighbor selection in multiresolution LBP (Ojala et al., 2002). Therefore we name this neighborhood system as multiresolution circular neighborhood system and represent by $(R, P)$ variable setting. In each resolution $(R, P)$, the model parameters are separately estimated. The $\alpha$ parameter represents the strength of interaction on center pixel by neighbors in a certain resolution and the $\sigma$ parameter corresponds to the roughness of the texture. Bilinear interpolation is used to estimate the neighbor values at off grid positions similar to (Ojala et al., 2002).

For the problem of emphysema and lung tissue discrimination this model is more appropriate because the texture patterns involving the problem have less directional qualities and more isotropic qualities (see (Sørensen et al., 2013)). Therefore with a minimal computational cost, the benefits of statistical model based feature extraction can be applied on to the problem of emphysema discrimination. 


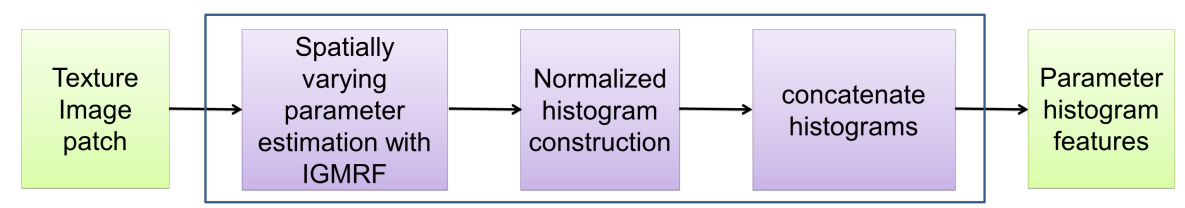

Figure 3: Parameter histogram texture feature formulation.

\subsection{Parameter Estimation}

The choice of using least squares estimation (LSE) for parameter estimation for GMRF is suggested by (Manjunath and Chellappa, 1991). LSE is used here for estimating parameters of the model. The main assumption behind the LSE method is that because (2) is Gaussian, the estimated value of $y_{s}$ is more probable to be the mean value of the function (Petrou and Sevilla, 2006). Therefore from the linear least square sense the residual will be,

$$
\varepsilon_{s}=y_{s}-\alpha \sum_{r \in N_{s}} y_{s+r}
$$

For least square fitting, given a stationary texture, sample neighborhoods of the texture are extracted by linear scanning of the region. Overlapping neighborhoods are also allowed (Li, 2009). When $N$ samples ( $\leq H * W$ ) of neighborhoods representing the texture is given the least square solution is,

$\alpha=\arg \min _{\alpha} \sum_{s \in \Omega} \varepsilon_{s}^{2}=\arg \min _{\alpha} \sum_{s \in \Omega}\left(y_{s}-\alpha \sum_{r \in N_{s}} y_{s+r}\right)^{2}$

By setting the first derivative to zero the parameter value can be obtained as,

$$
\alpha=\frac{\sum_{s \in \Omega}\left(y_{s} \sum_{r \in N_{s}} y_{s+r}\right)}{\sum_{s \in \Omega}\left(\sum_{r \in N_{s}} y_{s+r}\right)^{2}}
$$

The variance parameter of the model is then calculated by,

$$
\sigma^{2}=\frac{1}{N} \sum_{s \in \Omega}\left(y_{s}-\alpha \sum_{r \in N_{s}} y_{s+r}\right)^{2}
$$

The simple forms of solutions obtained for model parameters given by (5) and (6) can be easily implemented and efficiently computed.

\section{FEATURE FORMULATION}

Spatially varying parameter estimation is performed here to capture the structural and texel level local inhomogeneity of the texture. Spatially varying parameter estimation is sensitive to the subtle changes in the texture. (Dharmagunawardhana et al., 2012) have shown the significance in employing spatially varying parameters in model based statistical texture feature extraction and shown remarkable results on general texture segmentation. The local distributions of spatially varying parameters are better choice for discriminative texture features than using spatially constant GMRF parameters.

Localized small model estimation is performed here to estimate spatially varying parameters of the texture image or patch (Dharmagunawardhana et al., 2012). Small model estimation considers using local nearest neighborhood samples in the region $\Omega_{s}(\subset \Omega)$, for the estimation process. Here we use nearest five neighborhood samples, i.e $N_{k}, k \in$ $s+(0,0), s+(1,0), s+(-1,0), s+(0,1), s+(0,-1)$ or $k \in \Omega_{s}$ and $\|k-s\|_{2}=1$ (Figure 2). At each pixel, a localized estimation procedure is carried out similar to estimation method discussed in section 3.1, using these five nearest samples $(N=5)$. Then the estimated parameter values at pixel $s$ is,

$$
\alpha_{s}=\frac{\sum_{k \in \Omega_{s}}\left(y_{k} \sum_{r \in N_{k}} y_{k+r}\right)}{\sum_{k \in \Omega_{s}}\left(\sum_{r \in N_{k}} y_{k+r}\right)^{2}}
$$

The variance parameter of the model is given by,

$$
\sigma_{s}^{2}=\frac{1}{N} \sum_{k \in \Omega_{s}}\left(y_{k}-\alpha_{s} \sum_{r \in N_{k}} y_{k+r}\right)^{2}
$$

Estimating the parameter vectors $\left[\alpha_{s}, \sigma_{s}\right]^{T}$, for all the pixels in $\Omega$ results in two parameter images corresponding to the two model parameters. The normalized histogram of each parameter image is then concatenated into one long vector which is used as the feature vector of the texture image. The number of bins and the bin range is manually fixed. Hereafter we refer to these features as parameter histogram features and their construction is graphically illustrated in figure 3.

This approach can be seen as the inclusion of structural information into a statistical model based technique. (Dharmagunawardhana et al., 2012) have clearly demonstrated that this technique significantly 
Table 1: Confusion matrices for three class classification problem involving the classes NT, CLE and PSE. LBP result is obtained from (Sørensen et al., 2010).

\begin{tabular}{|l|ccc|}
\multicolumn{5}{c}{ noInt } \\
\hline & NT & CLE & PSE \\
\hline NT & 37 & 19 & 3 \\
CLE & 10 & 40 & 0 \\
PSE & 4 & 2 & 53 \\
\hline
\end{tabular}

\begin{tabular}{|l|ccc|}
\multicolumn{4}{c}{ joint } \\
\hline & NT & CLE & PSE \\
\hline NT & 57 & 2 & 0 \\
CLE & 2 & 48 & 0 \\
PSE & 1 & 2 & 56 \\
\hline
\end{tabular}

\begin{tabular}{|l|ccc|}
\multicolumn{5}{c}{$L B P$} \\
\hline & NT & CLE & PSE \\
\hline NT & 55 & 0 & 4 \\
CLE & 1 & 49 & 0 \\
PSE & 2 & 1 & 56 \\
\hline
\end{tabular}

enhances the discriminative power of model based features, specifically for texture classification and segmentation purposes.

\section{RESULTS AND DISCUSSION}

To evaluate the novel texture feature performance on emphysema diagnosis and quantification we use the online emphysema dataset from (Sørensen et al., 2013) which has been also used in performance evaluation of LBP and filter based features (Sørensen et al., 2010). The database comprises 115 HRCT slices of size $512 \times 512$ and 168 of square patches of size $61 \times 61$ obtained from a subset of slices. The HRCT slices belong to a study group of 39 subjects including non smokers, smokers and smokers with chronic obstructive pulmonary disease.

Each slice is labeled according to the leading pattern of interest and severity by an experienced chest radiologist and a pulmonologist. The leading patterns are normal tissue (NT), centrilobular emphysema (CLE), paraseptal emphysema (PSE) and panlobular emphysema (PLE). The severity of each slice is classified as no emphysema (0), minimal (1), mild (2), moderate (3), severe (4) and very severe (5). Leading pattern of each patch is also available and there are 59 NT patches, 50 CLE patches and 59 PSE patches. In this study, only NT, CLE and PSE classes are used. However, clinical test results on PFT tests are currently unavailable for the dataset. Therefore, correlations of the results with emphysema index and diagnosis from visual inspection by experience radiologists are considered.

\subsection{Emphysema Classification}

First, the patch dataset is used in a classification framework to identify the discriminative ability of the texture features. (Sørensen et al., 2010) reported satisfactory classification performance for this dataset with joint LBP using the parameter setting $(R, P)=$ $\{(1,8),(2,16)\}$ and a region of interest (ROI) of size $31 \times 31$. Following (Sørensen et al., 2010), the present study also employs $31 \times 31$ ROIs extracted from each $61 \times 61$ patches. For histogram calculation

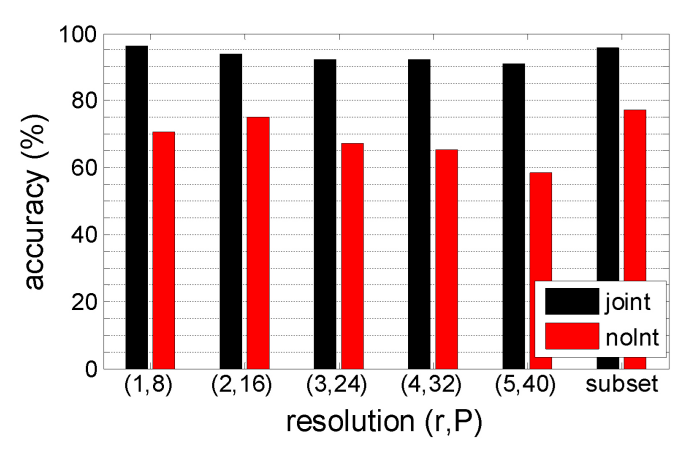

Figure 4: Leave one subject out classification accuracy for three class problem, NT,CLE and PSE.

bins $=\{10,20,30,40,50\}$ are evaluated and bins $=40$ is selected in the following experiments. The $\alpha$ parameter bin range is set from -1 to +1 . The $\sigma$ parameter bin range is set from 0 to 100 . The leave one subject out classification technique discussed in (Sørensen et al., 2010) with nearest neighbor classifier is used. In each leave one subject out trial, assigned labels are stored and in the end of all trials assigned labels are matched against the true labels to calculate the accuracy (Sørensen et al., 2010). The absolute sum of difference between histograms is taken as the distance metric.

The performance of parameter histograms with and without integrating intensity information is examined. The technique in which parameter histograms are constructed without integrating intensity information is referred to as 'noInt' and the joint intensity-parameter histograms are represented by 'joint' in this paper. Figure 4 shows the classification accuracies obtained for three class problem, NT, CLE and PSE for noInt and joint features with various neighborhood systems $(R, P)$. The accuracy gradually decreases with increasing resolution of the neighborhood system suggesting features from lower resolutions are more significant. The case 'subset' represents the integrated features from multiresolution levels of the neighborhood system $(R, P)=$ $\{(1,8),(2,16),(3,24)\}$. Based only on texture information, an accuracy of $77.4 \%$ can be obtained for the case 'subset'. However, integrating intensity vastly improves the accuracy to $95.8 \%$. This is comparable with the accuracies reported for LBP features which is $79.2 \%$ with only texture information and $95.2 \%$ with 

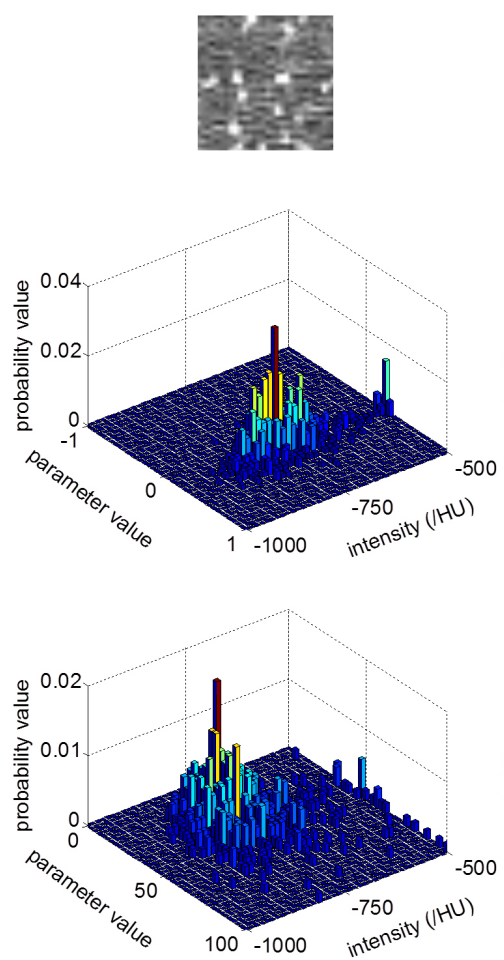

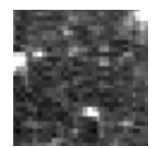

(a)

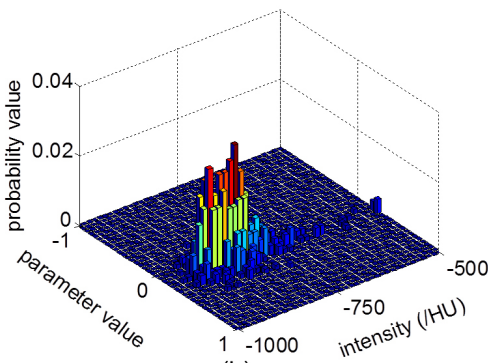

(b)

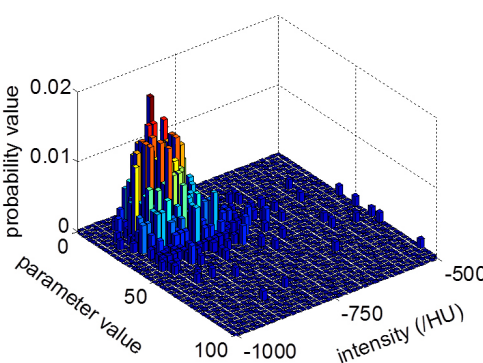

(c)
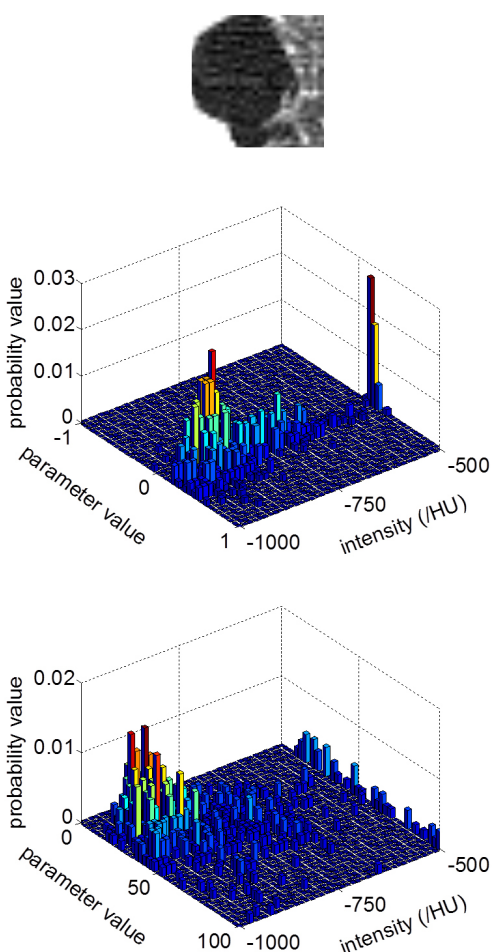

Figure 5: IGMRF parameter histograms. (a) original patches from the three classes NT, CLE, PSE respectively $(31 \times 31)$, (b) $\alpha$ and intensity joint histograms, (c) $\sigma$ and intensity joint histograms. $(R, P)=(1,8)$ and bins $=40$ in each axis are used.

Table 2: Comparison with other GMRF model based texture feature extraction methods.

\begin{tabular}{lccc}
\hline & joint & PL & TGMRF \\
\hline accuracy (\%) & 94.6 & 94.0 & 62.5 \\
time (s) & 16.1 & 50.2 & 3.09 \\
\hline
\end{tabular}

joint intensity-LBP features (Sørensen et al., 2010).

The confusion matrices obtained for the three class problem by the proposed features and LBP method are shown in table 1 . The LBP results are taken from the joint intensity-LBP histogram performance reported in (Sørensen et al., 2010). The confusion matrix of noInt features clearly indicate that NT and CLE classes have higher number of mis-classified ROIs as a consequence of disregarding intensity information. The joint parameter histogram features and joint LBP-intensity features perform comparatively well in discriminating different lung tissues.

Next the performance with other GMRF based texture features is evaluated for comparison. Table 2 shows the classification accuracies for joint parameter histogram features proposed here with $(R, P)=$ $(1,8)$ and bins $=20$, parameter local histogram features (PL) obtained from the technique in (Dharmagunawardhana et al., 2012) with second order neighborhood system and bins $=20$ and spatially con- stant GMRF features (TGMRF) based on (Manjunath and Chellappa, 1991) with second order neighborhood system. The joint parameter histogram, PL features and TGMRF features have a dimensionality of $R^{2 * b i n s^{2}}, R^{5 * b i n s^{2}}$ and $R^{5}$ respectively. The total time elapsed for feature extraction of complete patch dataset in Matlab R2013a environment running on a $2.67 \mathrm{GHz}$ CPU is also provided. Here, PL and TGMRF features are integrated with intensity similar to the joint parameter features.

It can be clearly seen that spatially varying parameters are more discriminative compared to spatially constant features obtained from TGMRF. However PL is comparatively slower because, firstly the model associates many parameters to estimate and secondly the subsequent histogram construction stage require more memory and computations. Compared to PL, the joint parameter histogram features provides faster feature formulation. The accuracies for joint parameter histogram features and PL are almost similar implying that for this specific problem IGMRF is sufficient and additional directional information in GMRF based PL features have not been of much use.

Therefore, based on classification performances, joint feature of IGMRF parameter histograms are a preferable efficient choice for emphysema and its sub- 


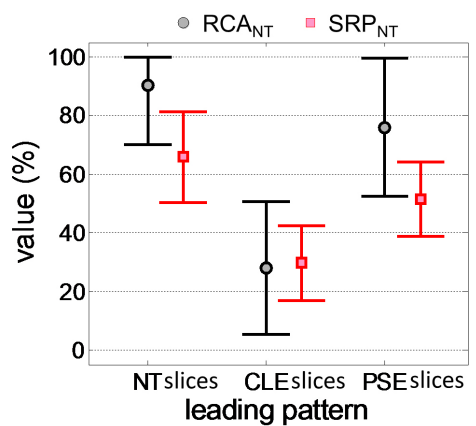

(a)

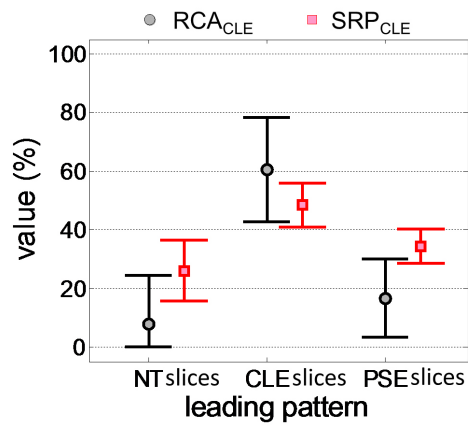

(b)

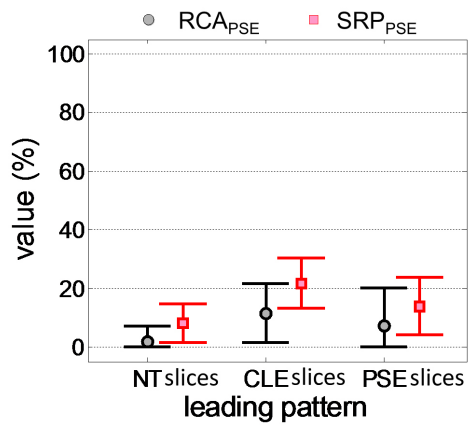

(c)

Figure 6: Tissue quantification with $R C A_{\omega_{c}}$ and $S R P_{\omega_{c}}$ for each category of slices (NT, CLE and PSE) grouped according to leading patterns labeled by visual inspection judgments. (a) NT tissue quantification, (b) CLE tissue quantification, (c) PSE tissue quantification. Note that the error bars are truncated near $0 \%$ and $100 \%$.

type diagnosis. Figure 5 shows the joint features of $\alpha$ and $\sigma$ parameters of IGMRF. Results could be further improved by feature selection.

\subsection{Emphysema Quantification}

In this section, we perform lung parenchyma pixel classification of $115 \mathrm{CT}$ slices for emphysema quantification. The features extracted from $31 \times 31$ labeled ROIs in section 5.1 are employed as the training models. The training models obtained from a subset of PSE ROIs are used. This subset represent moderate to severe PSE and clearly represent reasonably large regions of PSE tissue pattern with minimum confusion with NT or CLE class tissue. These PSE ROIs contains approximately $15 \%$ or less near boundary non parenchymal pixels. This setting of ROIs of PSE class can employ the prior information that PSE has high probability of occurrence near the boundary of the lung parenchyma (Sørensen et al., 2010). During the slice pixel classification all the training patches belong to the subject of corresponding slice are left out from training set.

The $(R, P)=(1,8)$ is used with bins $=40$ to construct joint parameter histogram features. The 6 slices out of 115 slices which belongs to class PLE are not considered because the patch data is unavailable. The remaining 109 slices with leading patterns NT, CLE and PSE are employed for tissue quantification. Note that the leading pattern of each slice is available prior to the quantification based on the visual inspection of experienced radiologists.

First of all, the spatially varying parameters of the lung parenchyma pixels are estimated. Then a window of size $31 \times 31$ is column-wise scanned on the parameter images and the normalized parameter histograms for each pixel is constructed.

The pixels outside the lung parenchyma is directly labeled as the background class by thresholding. The thresholding is based on the knowledge that the CT density values of lung parenchyma pixels are usually between $-1000 \mathrm{HU}$ to $-500 \mathrm{HU}$.

Hard and soft classification are performed here. The hard classification assigns each pixel a class label depending on the nearest training model to its feature vector. The soft classification finds the probability a pixel belongs to a certain class. We define the following expression to calculate the class probabilities.

$$
p\left(\omega_{c} / y_{s}\right)=\frac{\exp \left\{-D\left(h_{s}, M_{\omega_{c}}\right)^{2}\right\}}{\sum_{c=1}^{C} \exp \left\{-D\left(h_{s}, M_{\omega_{c}}\right)^{2}\right\}}
$$

where $\omega_{c}$ represent the class and $C$ is the number of classes. $h_{s}$ is the feature vector of the considered parenchyma pixel and $M_{\omega_{c}}$ is the nearest feature vector in the training set to $h_{s}$ from class $\omega_{c} . D($.$) is the$ sum of absolute difference distance metric.

The tissue quantification is then carried out on each slice. Two measures are obtained for each slice by fusing the results of all the lung parenchyma pixels on it. The relative hard classification accuracy $R C A_{\omega_{c}}$ (Sørensen et al., 2010) gives the percentage of lung tissue belonging to the class $\omega_{c}$. Based on soft classification probabilities we define the soft relative probability $S R P_{\omega_{c}}$ for a class $\omega_{c}$ according to,

$$
S R P_{\omega_{c}}=\frac{1}{\left|\Omega_{L}\right|} \sum_{s \in \Omega_{L}} p\left(\omega_{c} / y_{s}\right)
$$

where $\Omega_{L}$ is the lung parenchyma area and $\left|\Omega_{L}\right|$ is the number of pixels in the lung parenchyma.

$R C A_{\omega_{c}}$ is a measure about the spatial extent of the tissue from class $\omega_{c}$. The $S R P_{\omega_{c}}$ measure introduced here is sensitive to the severe tissue damages localized in a smaller area which can not be quantified properly as a higher degree of tissue damage by $R C A_{\omega_{c}}$.

The CT slices are categorized into groups according to the leading pattern labellings given by the radiologists. Then the averages and standard deviations 
$\mathrm{NT}(0)$
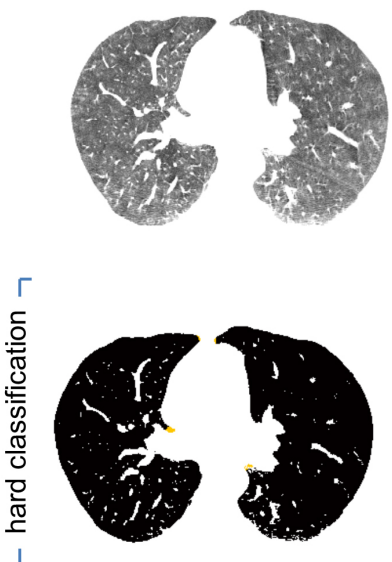

L
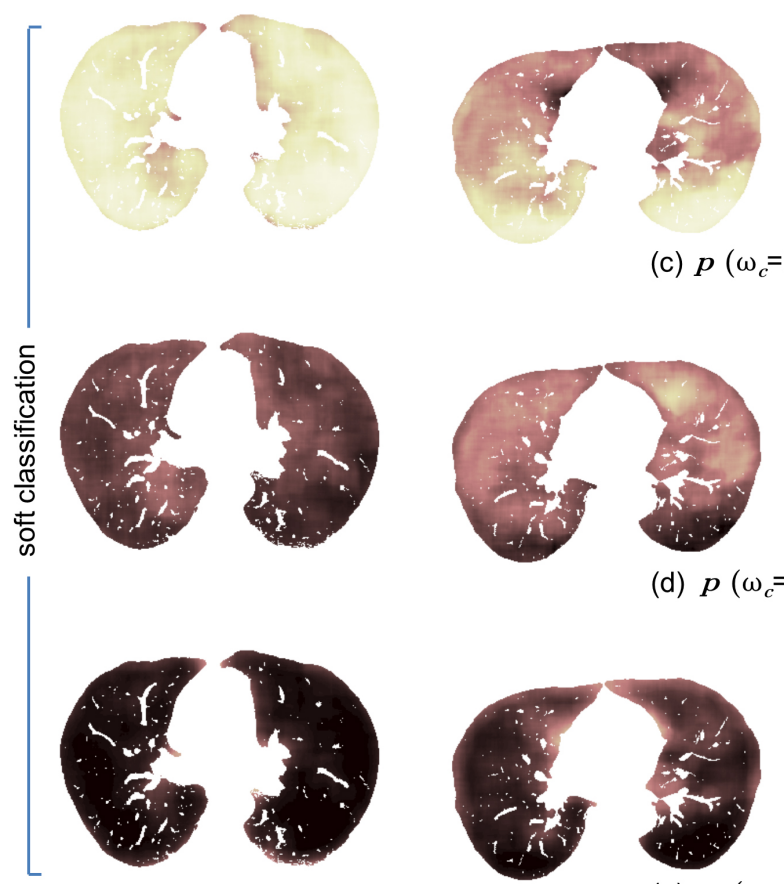

(c) $p\left(\omega_{c}=\mathrm{NT} / \mathrm{y}_{s}\right)$
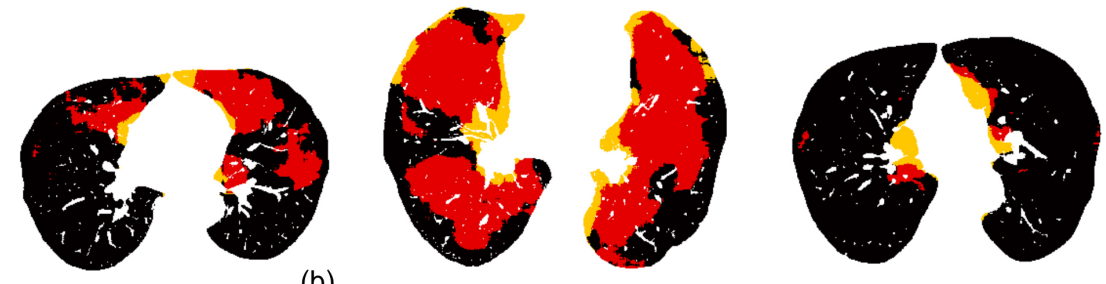

(b)
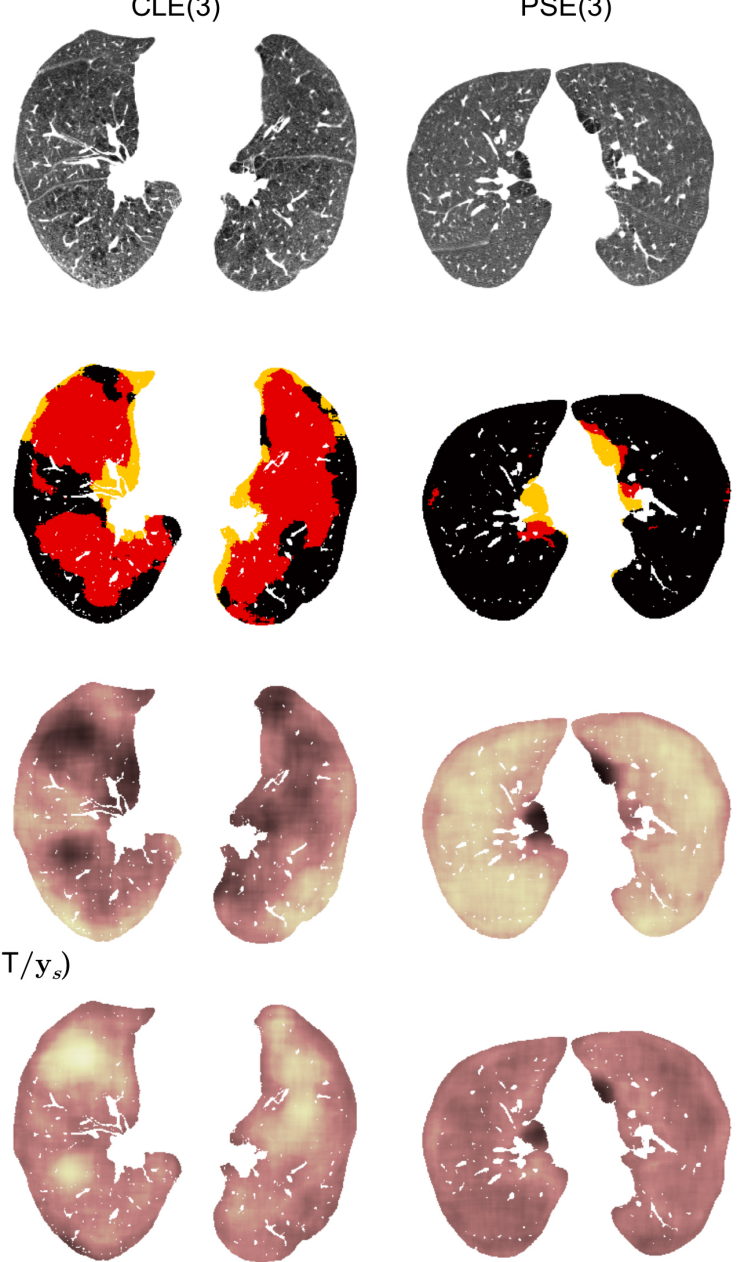

(d) $p\left(\omega_{c}=\mathrm{CLE} / \mathrm{y}_{s}\right)$
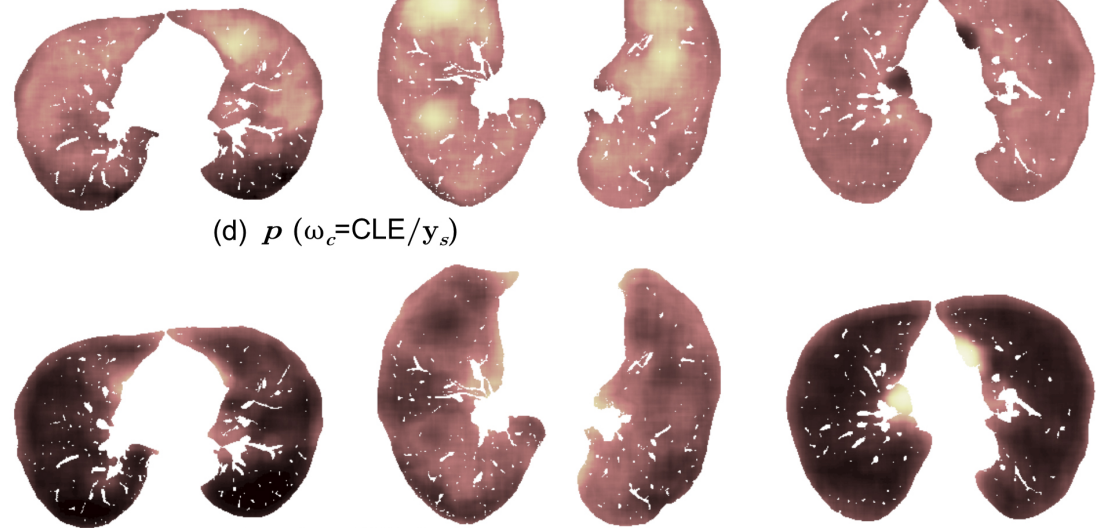

(e) $\boldsymbol{p}\left(\omega_{c}=\mathrm{PSE} / \mathrm{y}_{s}\right)$
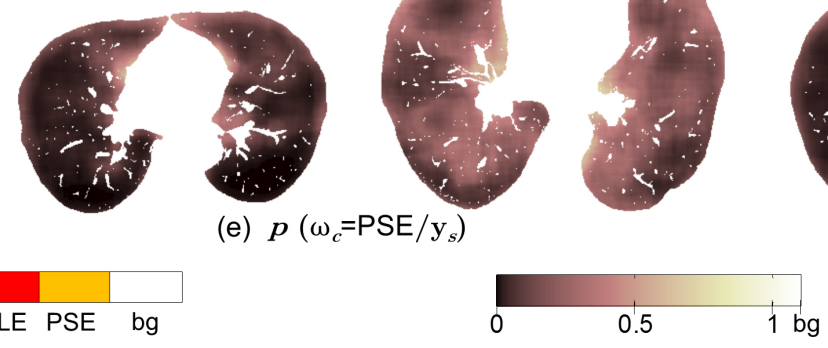

Figure 7: Lung parenchyma pixel classification for example slices representing NT, CLE and PSE slice groups. Severity is indicated in parenthesis. (a) original slices, (b) hard classification results, (c) $p\left(\omega_{c}=N T / y_{s}\right)$ (d) $p\left(\omega_{c}=C L E / y_{s}\right)$ and (e) $p\left(\omega_{c}=P S E / y_{s}\right)$ from soft classification. bg - background (pixels outside the lung parenchyma).

of $R C A_{\omega_{c}}$ and $S R P_{\omega_{c}}$ measurements of each catego ry is calculated separately. Results are shown in figure 6. The $R C A_{\omega c}$ and $S R P_{\omega_{c}}$ counts in figure 6 are consistent with the leading pattern labellings by visual inspections. For example, slices having leading pattern label as NT have a higher $R C A_{N T}$ and $S R P_{N T}$ counts and lower $R C A_{C L E}, R C A_{P S E}$ and $S R P_{C L E}, S R P_{P S E}$ counts. Most of the slices of PSE group in the considered slice dataset have minimal or mild PSE except for one severe PSE slice. Therefore $R C A_{N T}$ count is comparatively high indicating less tissue damage extent from PSE. However, it is relatively less than $R C A_{N T}$ for NT group.

Furthermore, figure $6 \mathrm{c}$ indicates that slices belong 


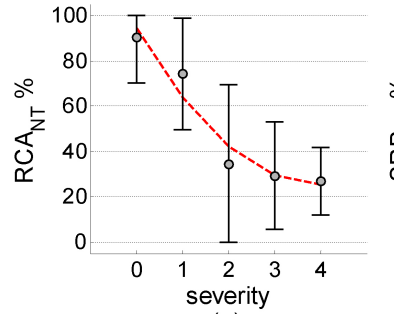

(a)

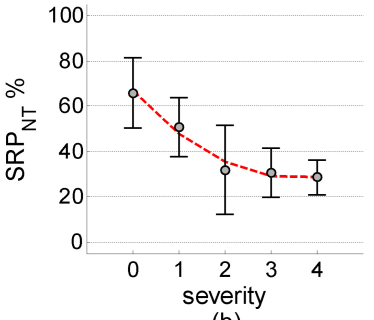

(b)
Figure 8: Tissue quantification with increasing severity.

to CLE group also have a tendency to contain higher value of PSE tissue. In real world situation, the CLE patients have a higher chance of co-existence of CLE and PSE. The slices belonging to PSE class have a relatively higher mis-classifications with CLE tissue giving higher $R C A_{C L E}$ and $S R P_{C L E}$ compared to $R C A_{P S E}$ and $S R P_{P S E}$ (figure $6 \mathrm{~b}$ and $\mathrm{c}$ ). $R C A_{\omega_{c}}$ has comparatively larger standard deviations (figure 6). This explains the relatively low sensitivity of $R C A_{\omega_{c}}$ compared to $S R P_{\omega_{c}}$ for pathology quantification.

Figure 7 demonstrates some example lung parenchyma labeled by using the proposed approach. The pathology distribution is effectively expressed. Texture is a regional property, therefore, hard classification achieves relatively larger areas of emphysematous tissue than from an intensity based thresholding technique like emphysema index (EI) (Muller et al., 1988). However, the correlation between total emphysema tissue count $\left(R C A_{C L E}+R C A_{P S E}\right)$ from proposed method and EI with $-910 H U$ threshold is large, giving a correlation coefficient of $0.84(p<$ $\left.10^{-4}\right)$. Moreover, pixels belonging to PSE tissue are often classified near the boundary of the lung parenchyma agreeing to the PSE location dependency near boundary (Figure 7b). The tendency that the CLE subjects also have PSE is also clearly reflected.

Finally, the severity of the slices and their corresponding tissue percentages are evaluated. The NT tissue counts $R C A_{N T}$ and $S R P_{N T}$ are shown in figure 8 . The severity level 5 only has $2 \mathrm{CT}$ slices and is not considered here. The $R C A_{N T}$ and $S R P_{N T}$ measures in figure 8 gradually reduce with increasing severity. Therefore the results in figure 8 have a good correlation with the severity labellings of the slices obtained by visual inspection.

\section{CONCLUSIONS}

We have performed emphysema quantification using a novel texture feature which integrates the essence of model based statistical and structural texture features. The online available emphysema dataset (Sørensen et al., 2013) is used here. The proposed texture feature is used in emphysema classification involving the classes NT, CLE and PSE. These results are comparable with the state of the art texture based LBP emphysema classification. We have also performed supervised lung paremchyma pixel classification for tissue quantification. The results illustrate convincing pathology distributions and successful quantification of lung tissues, well correlating with the class and severity labellings by visual inspection. $S R P_{\omega_{c}}$ measure obtained from soft classification has a higher sensitivity to emphysema pathology quantification than $R C A_{\omega_{c}}$. Evaluations on other emphysema datasets and clinical test data are considered in future. Also feature selection can be greatly helpful to reduce the higher dimensionality of the features. Nevertheless proposed feature perform efficient and effective lung tissue classification.

\section{ACKNOWLEDGEMENTS}

Authors gratefully acknowledge Dr. Lauge Sørensen for his kind support and all other researchers who contributed to making available the online computed tomography emphysema database. We are also immensely thankful to anonymous reviewers for their helpful comments.

\section{REFERENCES}

Coxson, H. O., Rogers, R. M., Whittall, K. P., Yachkova, Y. D., Par, P. D., Sciurba, F. C., and Hogg, J. C. (1999). A quantification of the lung surface area in emphysema using computed tomography. 159 (3):851-6.

Depeursinge, A., Iavindrasana, J., Hidki, A., Cohen, G., Geissbuhler, A., Platon, A., Poletti, P., and Müller, H. (2010). Comparative performance analysis of state-ofthe-art classification algorithms applied to lung tissue categorization. Journal of digital imaging, 23(1):1830.

Depeursinge, A., Sage, D., Hidki, A., Platon, A., Poletti, P. A., Unser, M., and Muller, H. (2007). Lung tissue classification using wavelet frames. In Proc. IEEE Int'l conf. of the Engineering in Medicine and Biology Society, pages 6259-6262. IEEE.

Dharmagunawardhana, C., Mahmoodi, S., Bennett, M., and Mahesan, N. (2012). Unsupervised texture segmentation using active contours and local distributions of Gaussian Markov random field parameters. In Proc. British Machine Vision Conference, pages 88.188.11 .

Gangeh, M. J., Sørensen, L., Shaker, S. B., Kamel, M. S., Bruijne, M., and Loog, M. (2010). A texton-based approach for the classification of lung parenchyma in CT images. 13:595-602. 
Häme, Y., Angelini, E. D., Hoffman, E. A., Barr, R. G., and Laine, A. F. (2013). Robust quantification of pulmonary emphysema with a hidden Markov measure field model. In Proc. 10th IEEE Int'l Symposium on Biomedical Imaging, pages 382-385. IEEE.

Hara, T., Yamamoto, A., Zhou, X., Iwano, S., Itoh, S., Fujita, H., and Ishigaki, T. (2004). Automated detection system for pulmonary emphysema on 3D chest CT images. In Proc. of SPIE, pages 915-919.

Kashyap, R. L. and Khotanzad, A. (1986). A model-based method for rotation invariant texture classification. IEEE Trans. on Pattern Analysis and Machine Intelligence, (4):472-481.

Kim, N., Seo, J. B., Lee, Y., Lee, J. G., Kim, S. S., , and Kang, S. (2009). Development of an automatic classification system for differentiation of obstructive lung disease using hrct. Journal of digital imaging, 22(2):136-148.

Li, S. Z. (2009). Markov Random Field Modeling in Image Analysis. Springer-Verlag London Ltd, 3rd edn edition.

Litmanovich, D., Boiselle, P. M., and Bankier, A. (2009). CT of pulmonary emphysema - current status, challenges, and future directions. 19:537-51.

Madani, A., Keyzer, C., and AGevenois, P. (2001). Quantitative computed tomography assessment of lung structure and function in pulmonary emphysema. 18 (4):720-30.

Mahmoodi, S. and Gunn, S. (2011). Snake based unsupervised texture segmentation using Gaussian Markov random field models. In Proc. 18th IEEE Int'l Conf. Image Processing, pages 1-4.

Manjunath, B. S. and Chellappa, R. (1991). Unsupervised texture segmentation using Markov random field models. IEEE Trans. on pattern analysis and machine intelligence, 13(5):478-482.

Mishima, M., T. Hirai, H. I., Nakano, Y., Sakai, H., Muro, S., Nishimura, K., Oku, Y., Chin, K., Ohi, M., Nakamura, T., Bates, J. H. T., Alencar, A. M., and Suki, B. L. (1999). Complexity of terminal airspace geometry assessed by lung computed tomography in normal subjects and patients with chronic obstructive pulmonary disease. In Proceedings of the National Academy of Sciences, volume 96, pages 8829-8834.

Muller, N., Staples, C., and R. Miller, R. A. (1988). Density mask, an objective method to quantitate emphysema using computed tomography. 94:782-787.

Ojala, T., Pietikainen, M., and Maenpaa, T. (2002). Multiresolution gray-scale and rotation invariant texture classification with local binary patterns. IEEE Trans. on pattern analysis and machine intelligence, 24:971987.

Petrou, M. and Sevilla, P. G. (2006). Image Processing, Dealing with Texture. John Wiley \& Sons Ltd.

Rue, H. and Held, L. (2005). Gaussian Markov Random Fields; Theory and Applications. Chapman \& Hall/CRC.

Sluimer, I. C., Waes, P. F., Viergever, M., and Ginneken, B. (2003). Computer-aided diagnosis in high resolution CT of the lungs. 30:3081.
Sørensen, L., Shaker, S. B., and de Bruijne, M. (2010). Quantitative analysis of pulmonary emphysema using local binary patterns. IEEE Transactions on Medical Imaging, 29(2):559-569.

Sørensen, L., Shaker, S. B., and de Bruijne, M. (2013). Computed Tomography Emphysema Database. http://image.diku.dk/emphysema_database/.

Sprawls, P. (1995). The Physical Principles of Medical Imaging. Medical Physics Publishing, USA, 2nd edition.

Uppaluri, R., Hoffman, E., M.Sonka, Hartley, P. G., W.Hunninghake, G., and McLennan, G. (1999). Computer recognition of regional lung disease patterns. 160 (2):648-54.

Vasconcelos, V., Silva, J. S., Marques, L., and Barroso, J. (2010). Statistical textural features for classification of lung emphysema in CT images: A comparative study. In Information Systems and Technologies (CISTI), pages 1-5. IEEE.

Xu, Y., Sonka, M., McLennan, G., Guo, J., and Hoffman, E. A. (2006). MDCT-based 3-D texture classification of emphysema and early smoking related lung pathologies. 156 (1):25.

Zhao, Y., Zhang, L., Li, P., and Huang, B. (2007). Classification of high spatial resolution imagery using improved Gaussian Markov random-field-based texture features. IEEE Trans. on Geoscience and Remote Sensing, 45(5):1458-1468. 